\title{
Serological diagnosis with the Chlamydia Spot-IF test
}

\author{
P. T. MANNION, H. MALLINSON* and J. D. TREHARNE†
} Liverpool Public Health Laboratory, Fazakerley Hospital, Lower Lane, Liverpool L9 7AL and tSection of Virology,
Institute of Ophthalmology, Judd Street, London WC1H 9OS

\begin{abstract}
Summary. Using a set of sera for which full chlamydial micro-immunofluorescence results suggested a clear diagnosis, we have evaluated the Chlamydia Spot-IF test (bioMerieux), which allows a comparison of titres to Chlamydia trachomatis and C. psittaci antigens. A modification of the test in which the antigen slides were pre-treated with a monoclonal antibody to chlamydial lipopolysaccharide, improved its ability to differentiate infections with $C$. trachomatis from those with $C$. psittaci or $C$. pneumoniae.
\end{abstract}

\section{Introduction}

Serological confirmation of a deep-seated chlamydial infection is often based at present on appropriate clinical details and a four-fold rise in antibody titre, or a single high titre, in a test such as the complement fixation (CF) test. The CF test is genus-specific and only confirms the presumptive diagnosis of lymphogranuloma venereum (LGV) or ornithosis. ${ }^{1}$ Chlamydia pneumoniae (TWAR) infection does not always produce a positive result by $\mathrm{CF}$ and $C$. trachomatis (CT) serovars (other than LGV) may produce a positive CF result only if deep-seated infection such as pelvic inflammatory disease (PID) is present. The whole inclusion fluorescence (WIF) test also includes a genus-specific reaction and can give positive results with TWAR infections. ${ }^{1}$ This may cause difficulties in interpretation in that the seroprevalence of TWAR in the general population can be as high as $19.9 \%{ }^{2}$ and a positive WIF result may, therefore, be misinterpreted as confirming a clinically suspected CT infection (e.g., in a patient with symptoms suggestive of PID).

CT infections involving superficial sites far outnumber any other type of chlamydial infection. ${ }^{3}$ These infections are best diagnosed by culture or by antigen detection (ELISA, immunofluorescence), but with deep-seated CT infections or for screening for the late sequelae of such infections, such as infertility, these tests often give negative results and a more sensitive and specific serological test is required. Microimmunofluorescence (MIF) fulfils this role and will reliably detect deep-seated chlamydial infections. ${ }^{1}$ However, the technical complexities of MIF limit its availability and usage. Dependent on the choice of antigens included, the MIF test can distinguish

Received 24 Oct. 1990; revised version accepted 25 Jan. 1991. * Correspondence should be sent to Dr H. Mallinson. between the different species of chlamydia and also the different serovars of CT. ${ }^{4}$

The commercial availability of an immunofluorescent test based on representative $\mathrm{CT}$ and $C$. psittaci (CP) antigens prompted an evaluation of this test (the Chlamydia Spot-IF test; bioMerieux, Basingstoke, Hants) with a set of sera for which full MIF results and clinical details were available.

The Chlamydia Spot-IF test is presented as (PTFE)coated microscope slides, each with 10 wells covered with CT (L2 serotype) or CP (avian) elementary bodies. Fluorescein-labelled anti-human IgG conjugate and a positive control serum are also supplied. A positive serum produces a "starry sky" appearance. The interpretation of the titres obtained is based on experimental evidence $^{5}$ that suggests that, with chronic infection, CT and CP titres are approximately equivalent, whereas $a \geqslant 4$ fold difference between the titres suggests infection with the species showing the higher titre.

\section{Materials and methods}

\section{Study group}

Between Feb. 1986 and Jan. 1989, a full range of chlamydial MIF tests was performed at the Institute of Ophthalmology, London on sera from 58 patients investigated at Liverpool Public Health Laboratory. The MIF results of $35(61 \%)$ of these patients were considered diagnostically useful, i.e., showed a predominant reaction to CT, CP or TWAR. Stored sera from 24 of these 35 patients were available in sufficient quantity for further testing. On the basis of a clear diagnosis suggested by their MIF results, these 24 patients were assigned to one of three groups to be tested by the Chlamydia Spot-IF test: (1) 11 patients with CT infection (T1-11); (2) 10 patients with CP infection (P1-10); (3) three patients with TWAR infection (TW1-3). 


\section{C. trachomatis and C. psittaci Spot-IF test}

Sera were tested in accordance with the manufacturer's instructions. The CT and CP antigen slides were removed from the refrigerator and placed at room temperature for $10-15 \mathrm{~min}$ before opening. Doubling dilutions of sera and control serum (Chlamytrol, bioMerieux) were made in phosphate-buffered saline (PBS) and 20- $\mu$ l volumes of each test serum dilution, the control serum and a conjugate (PBS) control were placed on a CP and CT slide. The slides were placed in a pre-warmed moist chamber for 30 min at $37^{\circ} \mathrm{C}$ and were then washed in PBS for $10 \mathrm{~min}$.

Excess fluid was drained off the slides and $20 \mu \mathrm{l}$ of a 1 in 200 dilution of fluorescein-labelled anti-human IgG conjugate (Fluoline-G, bioMerieux) was applied to each well. The incubation and washing steps were then repeated, and the slides were dipped in distilled water and gently blotted to remove excess fluid. The mountant (Fluoprep) and coverslips were applied. The slides were stored in a cool dark area and were read by two independent observers (H.M., P.M.) the same day with a Zeiss standard RA fluorescent microscope at $\times 400$ magnification. Neither observer was aware of the previous MIF results. The end-point was taken as the last well in which the pattern of fluorescence was still distinct.

If the observers' results were discrepant, the slides were re-assessed until results concurred.
Pre-treatment of C. trachomatis and C. psittaci

Spot-IF slides with anti-chlamydial

lipopolysaccharide monoclonal antibody (anti-LPS $M A b)$

Each slide well was treated with $20 \mu$ l of anti-LPS (anti-LPS enzyme conjugate from the IDEIA test, Novo Biolabs Ltd). The slides were incubated for 30 min at $37^{\circ} \mathrm{C}$ in a pre-warmed moist chamber, washed in PBS for $10 \mathrm{~min}$ and then drained. Slides were used immediately in the Spot-IF test.

\section{Results}

Table I shows Chlamydia Spot-IF results for 20 patients whose clinical diagnoses matched their classification as cases of CT, CP or TWAR infection on the basis of previous MIF results. With only three exceptions (patients T8, P4, P5), antibody titres measured by the Spot-IF test were higher than those reported by MIF. In the Spot-IF test, antibody titres to $C T$ antigen were at least four-fold higher than titres to $\mathrm{CP}$ antigen in all eight patients considered to have been infected with CT. Although the general tendency was for titres against CP antigen to be higher, significant four-fold or greater differences in Spot-IF

Table I. Chlamydia Spot-IF results in patients diagnosed as having CT, CP and TWAR infections

\begin{tabular}{|c|c|c|c|c|c|c|c|c|c|c|c|}
\hline \multirow{4}{*}{$\begin{array}{l}\text { Case } \\
\text { no.* }\end{array}$} & \multirow{4}{*}{ Sex } & \multirow{4}{*}{ Age } & \multirow{4}{*}{$\begin{array}{l}\text { Illness (duration, } \\
\text { days) }\end{array}$} & \multicolumn{8}{|c|}{ Serum titres in } \\
\hline & & & & \multirow{3}{*}{ CFT } & \multicolumn{5}{|c|}{ MIF test } & \multicolumn{2}{|c|}{ Spot-IF test } \\
\hline & & & & & \multicolumn{3}{|c|}{ TRACH } & \multirow{2}{*}{ PSITT } & \multirow{2}{*}{ TWAR } & \multirow{2}{*}{ TRACH } & \multirow{2}{*}{ PSITT } \\
\hline & & & & & $\mathrm{A}-\mathrm{C}$ & D-K & LGV & & & & \\
\hline $\mathrm{T} 1$ & $\mathbf{F}$ & $\ldots$ & PID & 80 & 256 & 256 & 256 & 16 & 32 & 8192 & 2048 \\
\hline $\mathrm{T} 2$ & $\mathbf{F}$ & 19 & Infertility & 40 & 1024 & 4096 & 2048 & 256 & 256 & 8192 & 1024 \\
\hline T3 & $\mathbf{F}$ & 22 & Vaginal discharge & 320 & 64 & 256 & 16 & 16 & $<16$ & 8192 & 2048 \\
\hline $\mathrm{T} 4$ & $\mathbf{F}$ & 27 & $\begin{array}{l}\text { Vaginal discharge } \\
\text { ?Fitz-Hugh Curtis }\end{array}$ & 20 & 256 & 256 & 256 & 32 & 128 & 1536 & 384 \\
\hline T5 & $\mathbf{F}$ & 28 & Infertility & 80 & 256 & 512 & 256 & $<16$ & $<16$ & 1536 & 128 \\
\hline T6 & $\mathbf{F}$ & 39 & ?Chlamydial infection & $<20$ & 256 & 256 & 256 & $<16$ & $<16$ & 1024 & 128 \\
\hline T7 & $\mathbf{F}$ & 31 & PID & 40 & 64 & 64 & 64 & $<16$ & $<16$ & 768 & 32 \\
\hline T8 & $\mathrm{F}$ & 24 & Reactive arthritis & $<20$ & 64 & 64 & 64 & $<16$ & $<16$ & 64 & $<8$ \\
\hline P1 & $\mathbf{M}$ & 60 & $\begin{array}{r}\text { Pneumonia (10) } \\
(46)\end{array}$ & $\begin{array}{r}20 \\
320\end{array}$ & $\begin{array}{l}\cdots \\
<16\end{array}$ & $\begin{array}{l}\ldots \\
<16\end{array}$ & $\begin{array}{l}\cdots \\
<16\end{array}$ & $\ddot{1024}$ & $\ldots$ & $\begin{array}{r}256 \\
4096\end{array}$ & $\begin{array}{r}512 \\
8192\end{array}$ \\
\hline $\mathbf{P} 2$ & $\mathbf{F}$ & 48 & Chest infection (24) & 640 & $<16$ & $<16$ & $<16$ & 2048 & 256 & 4096 & 2048 \\
\hline P3 & $\mathbf{M}$ & 22 & PUO (22) & $\ldots$ & $<16$ & $<16$ & $<16$ & 64 & 16 & 512 & 512 \\
\hline P4 & $\mathbf{F}$ & 64 & Pneumonia (23) & 40 & $<16$ & $<16$ & $<16$ & 2048 & 128 & 128 & 384 \\
\hline P5 & $\mathbf{M}$ & 48 & Pneumonia (13) & 40 & $<16$ & $<16$ & $<16$ & 4096 & 64 & 64 & 128 \\
\hline P6 & $\mathrm{F}$ & 32 & Pneumonia & 80 & 16 & 64 & 64 & 1024 & 128 & 1024 & 2048 \\
\hline P7 & $\mathbf{M}$ & 43 & Fever, chest pain & 320 & 16 & 16 & $<16$ & 256 & 256 & 1024 & 512 \\
\hline P8 & $\mathbf{M}$ & 55 & Pneumonia (30) & 80 & $<16$ & $<16$ & $<16$ & 128 & 128 & 512 & 512 \\
\hline P9 & $\mathrm{F}$ & 82 & ?Psittacosis (24) & 1280 & $<16$ & $<16$ & $<16$ & 1024 & 1024 & 8192 & 12288 \\
\hline TW1 & $\mathbf{M}$ & 77 & Chest infection (18) & 640 & $<16$ & $<16$ & $<16$ & $<16$ & 256 & 4096 & 4096 \\
\hline TW2 & $\mathbf{M}$ & 44 & Viral illness & 80 & $<16$ & $<16$ & $<16$ & 256 & 4096 & 1024 & 2048 \\
\hline TW3 & $\mathbf{M}$ & 50 & Pneumonia (25) & 80 & $<16$ & $<16$ & $<16$ & $<16$ & 64 & 768 & 768 \\
\hline
\end{tabular}

- T1-8, C. trachomatis; P1-9, C. psittaci; TW1-3, TWAR (C. pneumoniae).

TRACH, $C$. trachomatis antigens; PSITT, $C$. psittaei antigens; TWAR, $C$. pneumoniae antigens. 
titres to CT and CP antigens were not seen in any sera from the nine cases of CP infection or three cases of TWAR infection tested.

In an attempt to reduce the incidence of serological cross-reaction that results from the presence of genusspecific LPS in both CT and CP antigen preparations, some sera were re-tested with CT and CP Spot-IF slides which had been pre-treated ("blocked") with anti-LPS MAb (figure). For CT cases, "blocking" with anti-LPS MAb caused little or no change in titre to the CT antigen, whereas any substantial titres to the $\mathrm{CP}$ antigen were dramatically reduced. Differences between CT and CP titres for this group of cases of CT infections were thus accentuated in sera from five out of eight patients, including patient T3 whose serum showed no significant difference without blocking. The differentiation in the remaining three CT cases whose CT/CP titres differed significantly in the test without blocking, did not improve in the blocking test. However, in sera from patients considered to have CP or TWAR infection, "blocking" with antiLPS MAb caused large decreases in titres to both CT and $C P$ antigens.

In four patients (table II) the diagnosis suggested by MIF testing was not in keeping with their clinical symptoms. For three of these patients (T9, T10, T11) Spot-IF test results (including improved resolution on blocking) were consistent with the diagnosis of CT infection supported by MIF. The fourth patient (P10) had a high CF titre consistent with active chlamydial disease; his clinical details suggested LGV. The SpotIF test results were not consistent with $C T$ infection and the MIF results clearly seemed to indicate a CP/ TWAR infection.

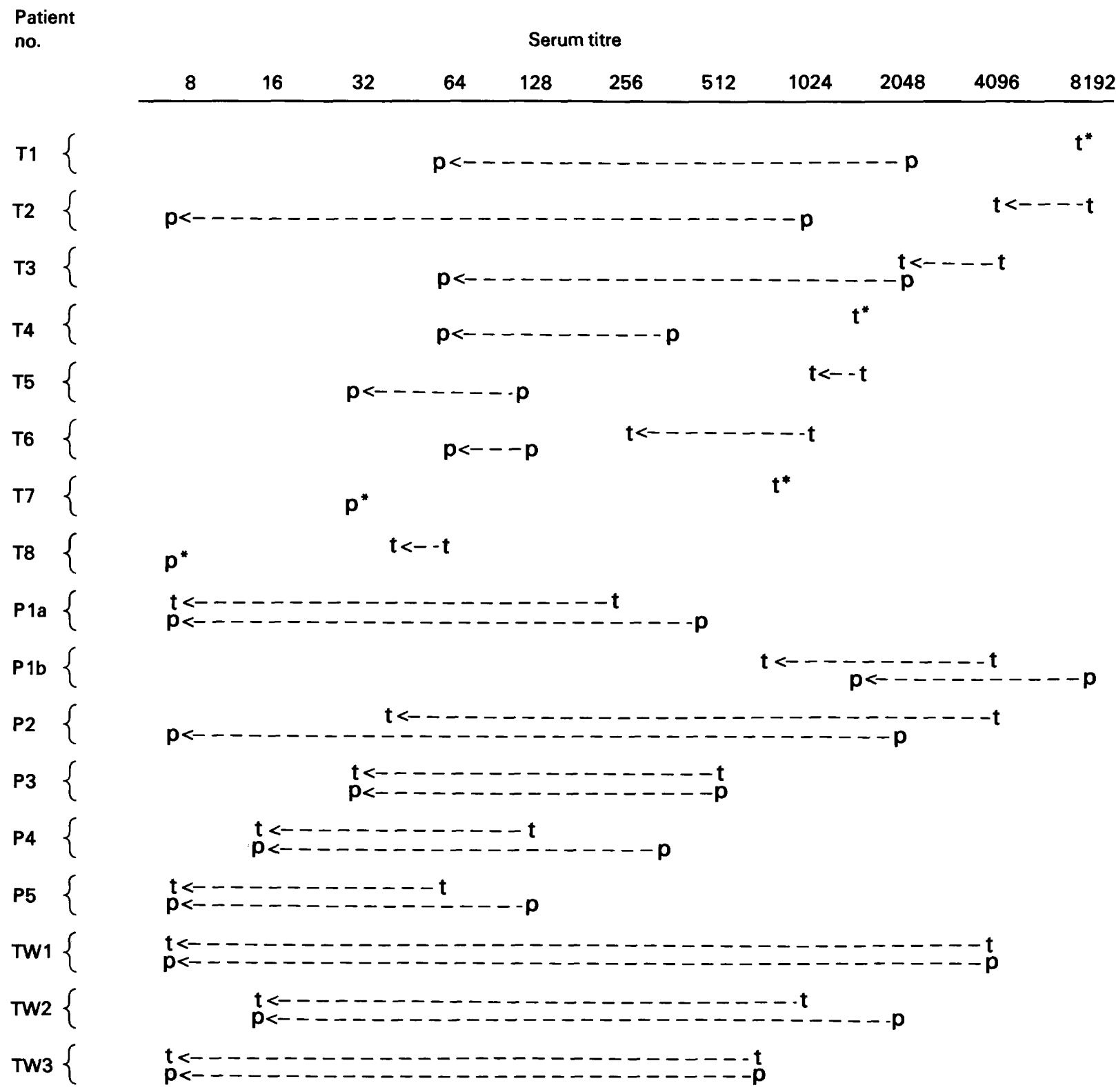

Figure. Reduction in antibody titre to $C$. trachomatis and C. psittaci after pre-treatment (blocking) of antigen slides with anti-LPS MAb. Titre with blocked slides $<-----$ titre with unblocked slides; $t, C$. trachomatis antigen slide; , $C$. psittacci antigen slide; ${ }^{*}$ titre unchanged after blocking. 
Table II. Chlamydia Spot-IF results in patients whose clinical illness and MIF results appeared contradictory

\begin{tabular}{|c|c|c|c|c|c|c|c|c|c|c|c|c|}
\hline \multirow{4}{*}{$\begin{array}{l}\text { Case } \\
\text { no. }\end{array}$} & \multirow{4}{*}{ Sex } & \multirow{4}{*}{ Age } & \multirow{4}{*}{ Illness (duration) } & \multicolumn{9}{|c|}{ Serum titres in } \\
\hline & & & & \multirow{3}{*}{ CFT } & \multicolumn{5}{|c|}{ MIF test } & \multicolumn{3}{|c|}{ Spot-IF test } \\
\hline & & & & & \multicolumn{3}{|c|}{ TRACH } & \multirow{2}{*}{ PSITT } & \multirow{2}{*}{ TWAR } & \multirow{2}{*}{$\begin{array}{l}\text { Antigen slide } \\
\text { pre-treatment }\end{array}$} & \multirow{2}{*}{ TRACH } & \multirow{2}{*}{ PSITT } \\
\hline & & & & & $A-C$ & D-K & LGV & & & & & \\
\hline T9 & $\mathbf{M}$ & 48 & $\begin{array}{l}\text { Pyrexia } \\
\text { Ex-Morocco }(18 \mathrm{~m})\end{array}$ & 40 & 64 & 128 & 128 & $<16$ & $<16$ & $\begin{array}{l}\text { PBS } \\
\text { anti-LPS }\end{array}$ & $\begin{array}{l}1024 \\
\text { ND }\end{array}$ & $\begin{array}{l}128 \\
\text { ND }\end{array}$ \\
\hline $\mathrm{T} 10$ & $\mathbf{F}$ & 54 & $\begin{array}{l}\text { Meningitis } \\
\text { (17d) }\end{array}$ & 40 & 128 & 128 & 64 & $<16$ & $<16$ & $\begin{array}{l}\text { PBS } \\
\text { anti-LPS }\end{array}$ & $\begin{array}{r}256 \\
256\end{array}$ & $\begin{array}{l}64 \\
32\end{array}$ \\
\hline $\mathrm{T} 11$ & $\mathbf{F}$ & $\ldots$ & Fever, sore throat $(3 \mathrm{~m})$ & 40 & 64 & 256 & 128 & $<16$ & $<16$ & $\begin{array}{l}\text { PBS } \\
\text { anti-LPS }\end{array}$ & $\begin{array}{l}256 \\
256\end{array}$ & $\begin{array}{l}128 \\
<8\end{array}$ \\
\hline P10 & $\mathbf{M}$ & $\ldots$ & Groin abscess ?LGV & 640 & $<16$ & $<16$ & $<16$ & 2048 & 1024 & $\begin{array}{l}\text { PBS } \\
\text { anti-LPS }\end{array}$ & $\begin{array}{r}2048 \\
512\end{array}$ & $\begin{array}{r}3072 \\
512\end{array}$ \\
\hline
\end{tabular}

ND, not done.

See footnote \& table I.

\section{Discussion}

The optimal test at present for the serological diagnosis of deep-seated chlamydial infection is MIF, but the complexity of maintaining stock cultures of all the relevant chlamydial species and serovars limits its use to specialist centres.

The need for a species-specific test has been highlighted further by the recent interest in community-based infections with TWAR. ${ }^{6}$ Serological evidence of past TWAR infection may be present in up to $20 \%$ of the UK population. ${ }^{2}$ This is of relevance if genus-specific serological tests are used to screen for CT or CP infection. ${ }^{7}$

The Spot-IF test was used to examine a panel of sera for which clinical details and serological results were available. Sera from cases of CT infection were adequately differentiated by the Spot-IF test. This was so even when clinical details suggested that samples were taken at a late stage in the infection-a stage during which the manufacturers had predicted little difference in the CT/CP titres.

In cases of CP infection, the Spot-IF titres against both antigens were equivalent, with both early and late sera. Sequential sera available from three cases of $\mathrm{CP}$ infection showed rising titres ( $\geqslant 4$ fold) to both $\mathrm{CT}$ and $\mathrm{CP}$ antigens. Thus, the Spot-IF test seemed unable to confirm $\mathrm{CP}$ infections adequately. The same result was found with sera from the three cases of TWAR infection.

The presence of genus-specific LPS antigen is a useful attribute, allowing group antibody to be detected in a single CF test. However, LPS was considered to be a possible source of cross-reactivity in the Spot-IF test. Our results suggest that "blocking" of antigen slides with anti-LPS MAb may be a useful general approach to modulating such cross-reactivity in serological tests for chlamydia. Other workers have developed serological tests based on antigens treated chemically to remove the LPS. ${ }^{8}$ With the Spot-IF test, whole elementary bodies are the target antigens and are supplied fixed as ready prepared slides. Therefore, blocking with a commercial anti-LPS MAb seemed a logical approach.

The anti-LPS MAb that was used reacts with LPS from both CT and CP (TWAR not having been considered at that time). ${ }^{9}$ We cannot exclude the possibility that steric inhibition of other epitopes that are part of, or lie in close proximity to, the LPS may occur because of the attached enzyme. The response of sera from cases of CT infection would seem to suggest that the antibody responses are predominantly against antigens specific to CT (possibly major outermembrane protein-MOMP) with only a limited antiLPS response. In contrast, sera from cases of $\mathrm{CP} /$ TWAR infection seem to have an antibody directed predominantly against genus-specific chlamydial LPS. This could relate to the strain of C.psittaci used in the Spot-IF test. It is likely that there is marked antigenic diversity within what is now classified as the $C$. psittaci species, and the single CP isolate used in the spot-IF test may not share many of the surface-exposed epitopes expressed by other CP strains.

This diversity of response may also relate to the timing of the serum samples. Onset dates were available for most cases of CP/TWAR infection and all the samples were obtained within 6 weeks of onset. Unfortunately, precise dates of onset were not available for the cases of CT infection, but clinical details suggested that the majority of specimens were from late in the course of the illness. Hence an initial genusspecific response could occur in all chlamydial infections, to be overtaken by a species-specific response later in the illness. Alternatively, our results would be consistent with a predominantly species-directed response in cases of $\mathrm{CT}$ infection and a genus-specific response in cases of CP/TWAR infection that persisted throughout the infection.

One group of workers has suggested that LPS may be lost from CT elementary bodies by the effect of 
antibody binding, as a protection from immune destruction. ${ }^{10}$ Other workers have demonstrated that cells infected with CT have chlamydia-specific LPS associated with their plasma membrane. ${ }^{11}$ Therefore, LPS may be the more prominent immunogen early in CT infections, whereas antibodies against MOMP develop later in the infection. However, this would be contrary to observations of a genus-specific response appearing later than the initial species-specific response during hyper-immunisation of experimental animals. $^{12}$

In summary, with sera for which the full MIF test gave diagnostically useful information, the Spot-IF

\section{References}

1. Barnes RC. Laboratory diagnosis of human chlamydial infections. Clin Microbiol Rev 1989; 2: 119-136.

2. Forsey T, Darougar S, Treharne JD. Prevalence in human beings of antibodies to Chlamydia IOL-207, an atypical strain of chlamydia. $J$ Infect 1986 ; 12: 145-152.

3. Treharne JD, Ballard RC. The expanding spectrum of the Chlamydia - a microbiological and clinical appraisal. Rev Med Microbiol 1990; 1: 10-18.

4. Wang S-P, Grayston JT, Alexander ER, Holmes KK. Simplified microimmunofluorescence test with trachoma-lymphogranuloma venereum (Chlamydia trachomatis) antigens for use as a screening test for antibody. J Clin Microbiol 1975; 1: $250-255$.

5. Orfila J, Eb F. Serodiagnosis in chlamydial respiratory infection of the newborn. Isr J Med Sci 1983; 19: 940-942.

6. Grayston JT. Chlamydia pneumoniae, strain TWAR. Chest $1989 ; 95: 664-669$.

7. Bourke SJ, Carrington D, Frew CE, Stevenson RD, Banham $\mathrm{SW}$. Serological cross-reactivity among chlamydial strains test in its basic format confirmed deep-seated CT infections. In the "modified" form of the test the predominant reaction in CT infections was accentuated and cases of CP/TWAR infection showed a consistent (if unexpected) pattern of results. Further work is needed to assess more fully the range of titres that represent past or recent infection. The test, in its modified format, may be of help in laboratories that currently rely on a genus-specific test for the serological confirmation of deep-seated chlamydial infection.

We thank Dr K. J. Mutton, Consultant Virologist, for providing sera from suitable patients and Miss Karen Whalley for secretarial assistance.

in a family outbreak of psittacosis. J Infect 1989; 19: 4145.

8. Ladany S, Black CM, Farshy CE, Ossewaarde JM, Barnes RC. Enzyme immunoassay to determine exposure to Chlamydia pneumoniae (strain TWAR). J Clin Microbiol 1989; 27: 2778-2783.

9. Thornley MJ, Zamze SE, Byrne MD, Lusher M, Evans RT. Properties of monoclonal antibodies to the genus-specific antigen of Chlamydia and their use for antigen detection by reverse passive haemagglutination. J Gen Microbiol $1985 ; 131: 7-15$.

10. Birkelund $S$, Lundemose AG, Christiansen G. Immunoelectron microscopy of lipopolysaccharide in Chlamydia trachomatis. Infect Immun 1989; 57 : 3250-3253.

11. Karimi ST, Schloemer RH, Wilde CE. Accumulation of chlamydial lipopolysaccharide antigen in the plasma membranes of infected cells. Infect Immun 1989; 57: 17801785.

12. Wang S-P, Grayston JT. Local and systemic antibody response to trachoma eye infection in monkeys. In: Nichols RL (ed) Trachoma and related disorders (International Congress Series No. 223). Amsterdam, Excerpta Medica. 1971: 216232 . 